\title{
Cross sections and forward multiplicities measurements with TOTEM
}

\author{
Mirko BERRETTI ${ }^{* \dagger}$ \\ (University of Siena and Pisa INFN (IT)) \\ E-mail: mirko.berretti@cern.ch
}

The TOTEM experiment at the LHC has performed the first luminosity-independent measurements of the total proton-proton cross-section at $\sqrt{s}=7 \mathrm{TeV}$ and $8 \mathrm{TeV}$. These measurements are based on the optical theorem and require simultaneous measurements of the elastic rate by measuring the outgoing protons with Roman Pots and of the inelastic rate, accomplished with the forward charged-particle telescopes T1 and T2 in the range 3.1 $<|\eta|<6.5$. TOTEM has also determined the total pp cross-section using the CMS luminosity measurement in various ways and the results were found in excellent agreement with the luminosity-independent measurement, despite having very different systematic dependencies. Moreover, since TOTEM is capable to detect inelastic events with diffractive masses down to $3.4 \mathrm{GeV}$, a limit on the cross section for low mass diffraction was set from the difference between the overall inelastic cross section (measured only using elastic scattering) and visible inelastic cross section (measured using T1 and T2). The differential elastic cross section has been measured in the range $5 \cdot 10^{-3}<|t|<2.5 \mathrm{GeV}^{2}$ for pp collisions at the center of mass energy of $7 \mathrm{TeV}$ and down to $|t|=0.01 \mathrm{GeV}^{2}$ for collisions at $8 \mathrm{TeV}$. The measurement of the forward charged particle $\eta$-density has been performed at 7 $\mathrm{TeV}$ in the range $5.3<|\eta|<6.4$. This measurement constitutes the most forward pseudorapidity density measurement ever obtained at LHC. This analysis is also repeated at $8 \mathrm{TeV}$ by using a dedicated run taken with a common CMS/TOTEM trigger. A summary of these measurements is here reported, as well as the preliminary results on single and double diffractive cross section.

XXI International Workshop on Deep-Inelastic Scattering and Related Subject 22-26 April 2013

Marseilles,France

\footnotetext{
* Speaker.

† On behalf of the TOTEM Collaboration
} 


\section{The TOTEM experiment}

The TOTEM Experiment at the LHC [1] is designed to measure the total proton-proton crosssection $\left(\sigma_{t o t}\right)$ with a precision down to $1-2 \%$, to study the differential elastic cross section $\left(d \sigma_{e l} / d t\right)$ over a wide range of the squared four-momentum transfer and to make measurements on diffractive processes, partially in cooperation with the CMS experiment [2]. The experimental apparatus is sketched in fig. 1.

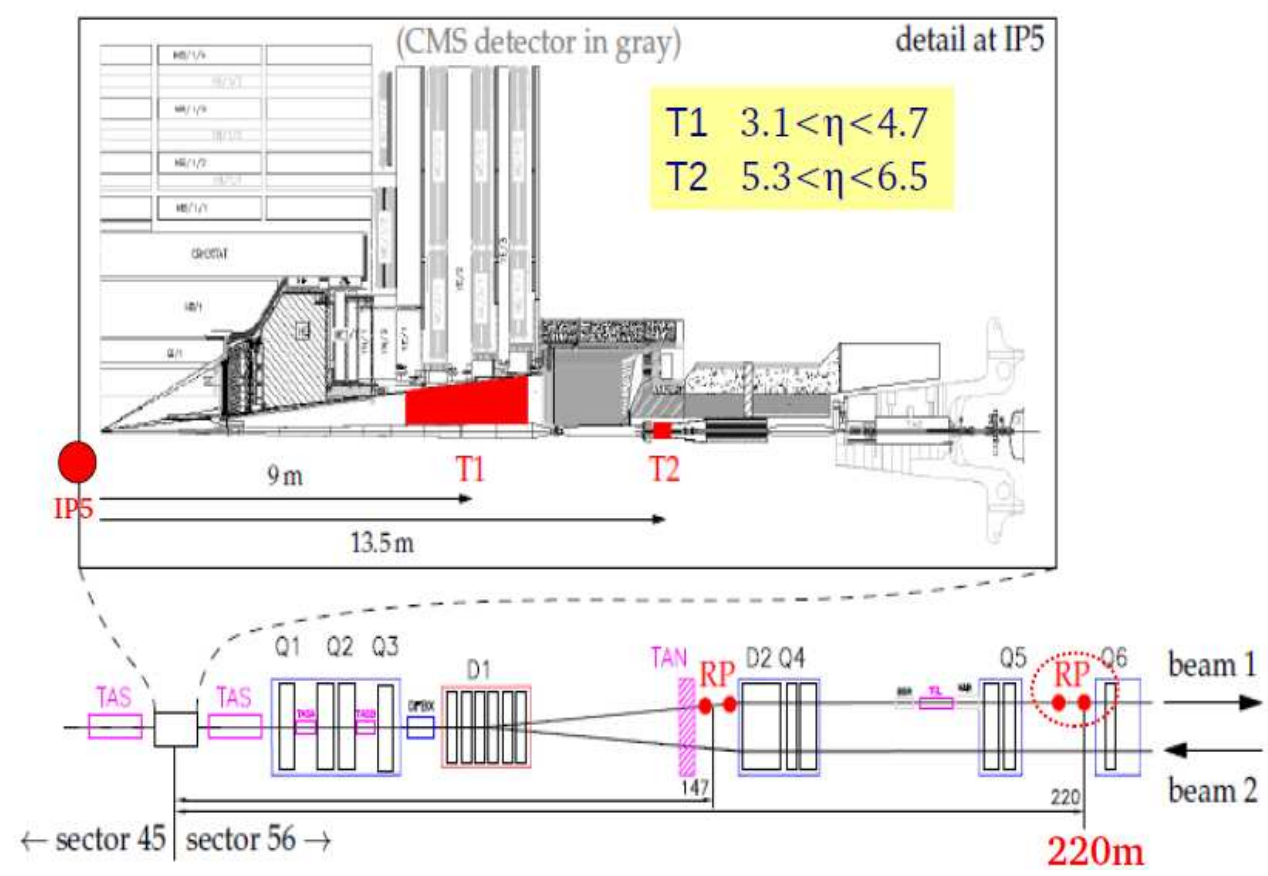

Figure 1: Top: the T1 and T2 telescopes in the forward region of CMS. Bottom: the Roman Pots location along the LHC beam-line. All TOTEM detectors are installed symmetrically with respect to the IP 5.

To achieve optimum forward coverage for charged particles emitted by the pp collisions in the interaction point IP5, two tracking telescopes, T1 and T2, covering respectively the pseudorapidity range $3.1<|\eta|<4.7$ and $5.3<|\eta|<6.5$, are installed on each side. Special movable beam-pipe insertions - called Roman Pots (RP) - are placed at distances of $\sim 147 \mathrm{~m}$ and $\sim 220 \mathrm{~m}$ from IP5 and allow the measurement of the leading proton coming from diffractive or elastic interactions.

\section{Total, elastic and inelastic $p p$ cross section}

TOTEM performed a first measurement of $\mathrm{d} \sigma_{e l} / \mathrm{dt}$ at $\sqrt{s}=7 \mathrm{TeV}$ in the $0.36<|t|<2.5 \mathrm{GeV}^{2}$ range using data taken in 2010 with the standard LHC optics $\left(\beta^{*}=3.5 \mathrm{~m}\right)$, during a dedicated run at low luminosity [3]. In June 2011, with a dedicated beam optics configuration $\left(\beta^{*}=90\right.$ $\mathrm{m})$ and low luminosity, the elastic scattering rate $\left(N_{e l}\right)$ was measured down to $|t|=2 \cdot 10^{-2} \mathrm{GeV}^{2}$ [4], with $\sim 67 \%$ of the full elastic rate detected. With the use of the optical theorem, the CMS luminosity ( $\mathscr{L}$, known with $4 \%$ uncertainty) and the $\rho$ parameter ${ }^{1}$ estimated by the COMPETE [6]

\footnotetext{
${ }^{1} \rho=\Re\left|f_{e l}(0)\right| / \mathfrak{I}\left|f_{e l}(0)\right|$, where $f_{e l}(0)$ is the forward nuclear elastic amplitude.
} 
collaboration, the elastic and total cross section was determined:

$$
\sigma_{t o t}^{2}=\left.\frac{16 \pi(\hbar c)^{2}}{1+\rho^{2}} \cdot \frac{1}{\mathscr{L}} \frac{d N_{e l}}{d t}\right|_{t=0}
$$

The analysis was also repeated with a low luminosity run at $\beta^{*}=90 \mathrm{~m}$ taken in October 2011 where even smaller $|t|$ values, down to $5 \cdot 10^{-3} \mathrm{GeV}^{2}$, were measured. This enabled the observation of $91 \%$ of $\sigma_{e l}$, allowing an improved extrapolation to the optical point [5].

The TOTEM differential elastic cross-section measurements at $\sqrt{s}=7 \mathrm{TeV}$ are reported in figure 2, where the analysis results obtained in three different runs and $|t|$-intervals, are shown.

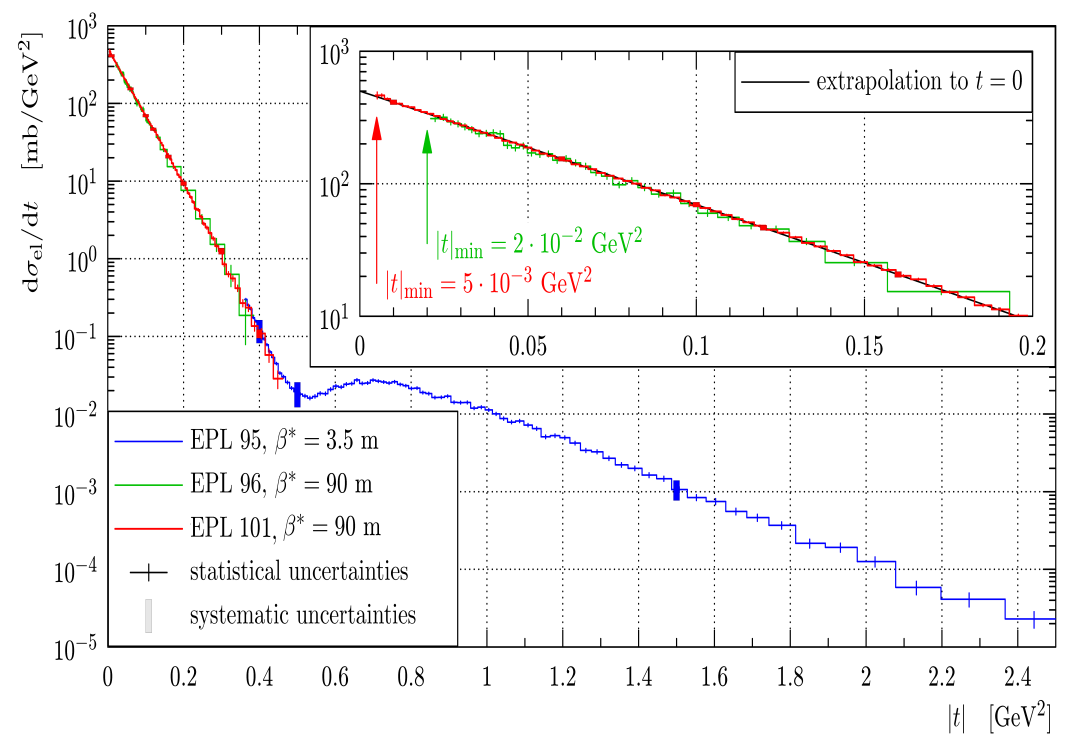

Figure 2: Elastic $|t|$-differential cross section at $\sqrt{s}=7 \mathrm{TeV}$.

For $|t|<0.2 \mathrm{GeV}^{2}$ the data can be described very well by an exponential function with slope $B=19.9 \pm 0.3 \mathrm{GeV}^{2}$. The diffractive minimum was found at $\left|t_{\min }\right|=0.53 \pm 0.01 \mathrm{GeV}^{2} . B$ and $\left|t_{\min }\right|$ are found to be respectively larger and smaller with respect to the values obtained from the elastic scattering measurements at lower-energy. Therefore with the increase of the center of mass energy the "shrinkage of the diffractive peak in elastic pp collision" was confirmed by TOTEM. In the $|t|$-range $1.5-2.0 \mathrm{GeV}^{2} d \sigma_{e l} / d t$ can be described by a power law $|t|^{n}$ with $\mathrm{n}=-7.8 \pm 0.3$. The luminosity-dependent total, elastic and inelastic cross section, obtained by measuring the elastic rate only, were found to be [5]:

$$
\sigma_{\text {tot }}=98.6 \pm 2.2 \mathrm{mb} \quad \sigma_{e l}=25.4 \pm 1.1 \mathrm{mb} \quad \sigma_{\text {inel }}=73.2 \pm 1.3 \mathrm{mb}
$$

With the same data as in [5], $\sigma_{\text {inel }}$ was also directly measured using inelastic events triggered by the T2 telescope, with the luminosity provided by CMS [7]. The inelastic cross section visible to T2 was found to be $69.73 \pm 2.88 \mathrm{mb}$. From the study of a sample triggered on bunch crossing the contribution of the $\mathrm{T} 1$ telescope to the inelastic rate was also determined. The cross-section for inelastic events with at least one $|\eta|<6.5$ final-state particle was determined to be $70.5 \pm 2.9 \mathrm{mb}$. This cross-section includes all central diffractive events of which maximally $0.25 \mathrm{mb}$ is estimated 
to escape the detection of the telescopes. The $\mathrm{T} 1+\mathrm{T} 2$ telescopes are expected to be sensitive to diffractive masses larger than $3.4 \mathrm{GeV}$. Based on models for low mass diffraction, the total inelastic cross-section was deduced to be $73.7 \pm 3.4 \mathrm{mb}$. An upper limit of $6.31 \mathrm{mb}$ at $95 \%$ confidence level on the cross-section for events with diffractive masses below $3.4 \mathrm{GeV}$ was obtained from the difference between the overall inelastic cross-section obtained by TOTEM using elastic scattering [5] and the cross-section for inelastic events with at least one $|\eta|<6.5$ final-state particle. By using the optical theorem the luminosity of the run used for the luminosity-independent measurement of the cross sections [8] was determined:

$$
\mathscr{L}_{\text {int }}=\frac{1+\rho^{2}}{16 \pi(\hbar c)^{2}} \cdot \frac{\left(N_{e l}+N_{\text {inel }}\right)^{2}}{d N_{e l} /\left.d t\right|_{t=0}}
$$

The value estimated by TOTEM $\left(83.7 \pm 3.2 \mu \mathrm{b}^{-1}\right)$ was in good agreement with the one provided by CMS $\left(82.8 \pm 3.3 \mu \mathrm{b}^{-1}\right)$. The measurement of the luminosity independent total cross section was obtained from the relation:

$$
\sigma_{t o t}=\frac{16 \pi(\hbar c)^{2}}{1+\rho^{2}} \cdot \frac{d N_{e l} /\left.d t\right|_{t=0}}{N_{e l}+N_{\text {inel }}}
$$

where $N_{e l}$ and $N_{\text {inel }}$ represent respectively the elastic and inelastic rates integrated over a given data taking period. The total, elastic and inelastic luminosity-independent cross section were found to be[8]:

$$
\sigma_{\text {tot }}=98.0 \pm 2.5 \mathrm{mb} \quad \sigma_{e l}=25.1 \pm 1.1 \mathrm{mb} \quad \sigma_{\text {inel }}=72.9 \pm 1.5 \mathrm{mb}
$$

The errors on the $\sigma_{e l}$ measurement are dominated by the uncertainties on the extrapolation to $t=0$ while the $\sigma_{\text {inel }}$ leading uncertainty is due to the correction for the contribution from low mass diffraction.

Using the same analysis procedures, the cross sections were also determined at $8 \mathrm{TeV}$ [9], in a low luminosity run with $\beta^{*}=90 \mathrm{~m}$ where the elastic proton $|t|$ was measured down to $\left|t_{\min }\right|=$ $10^{-2} \mathrm{GeV}^{2}$. The luminosity independent results are:

$$
\sigma_{\text {tot }}=101.7 \pm 2.9 \mathrm{mb} \quad \sigma_{e l}=27.1 \pm 1.4 \mathrm{mb} \quad \sigma_{\text {inel }}=74.7 \pm 1.7 \mathrm{mb}
$$

The cross section measurements at 7 and $8 \mathrm{TeV}$, in good agreement with the COMPETE extrapolation, are summarised in fig. 3 (left). The right part of fig. 3 reports a comparison of the cross sections measurements at $\sqrt{s}=7 \mathrm{TeV}$ obtained with the different analysis methods [8]: in $(1,2)$ the optical theorem, the luminosity from CMS and $\rho$ from the COMPETE fit were used, for two runs with different instantaneous luminosity and different position of minimum approach of the RPs to the beam; in (3) a $\rho$ independent measurement was performed, using the luminosity from CMS and the relation $\sigma_{t o t}=\sigma_{e l}+\sigma_{\text {inel }}$; in (4) the cross sections were evaluated with the luminosity independent method. It is important to remind that an elastic and total cross section analysis at $8 \mathrm{TeV}$ is also ongoing based on a low luminosity run with $\beta^{*}=1 \mathrm{~km}$ where the elastic proton $|t|$ was measured down to $\left|t_{\min }\right|=6 \cdot 10^{-4} \mathrm{GeV}^{2}$ and a detailed study on the Hadronic/Coulomb interference is performed. 


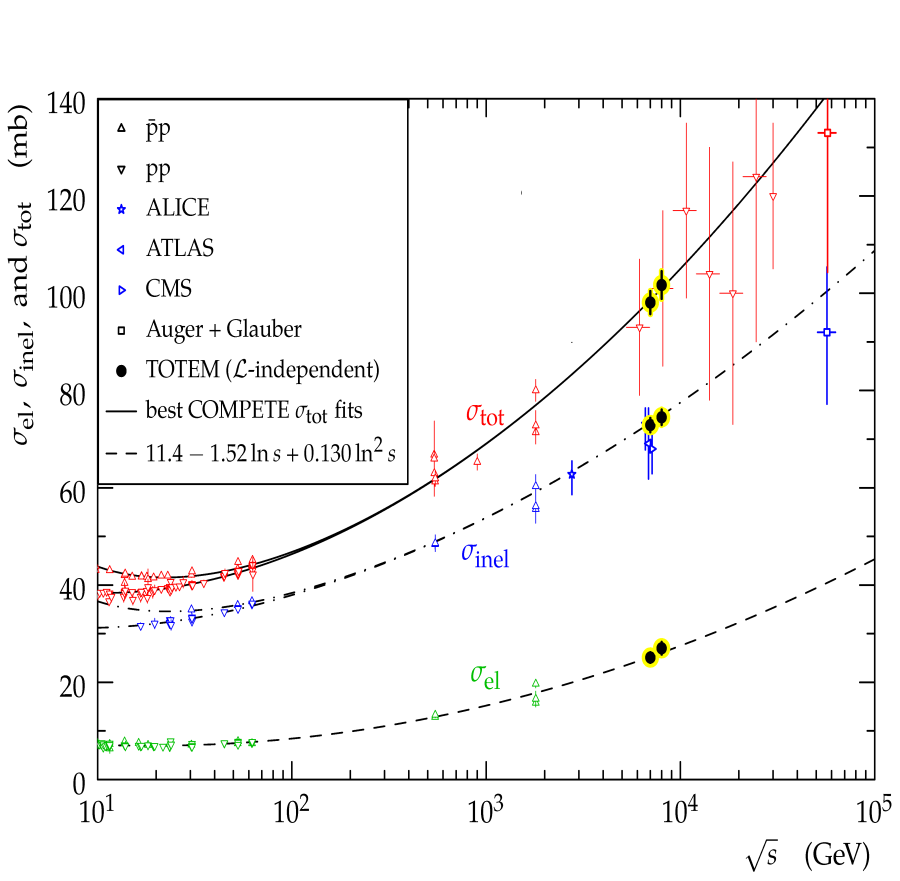

Measurements at $\sqrt{s}=7 \mathrm{TeV}$
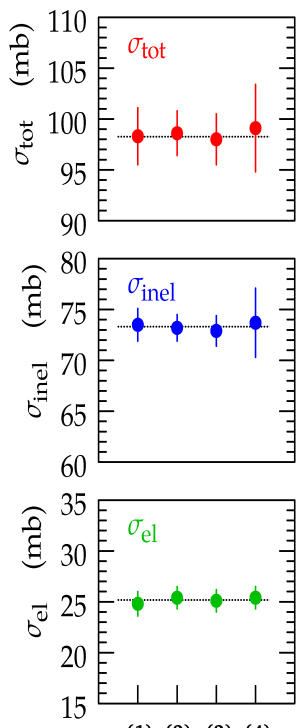

(1) (2) (3) (4)

Figure 3: Left: measurement of the luminosity-independent $\sigma_{t o t}, \sigma_{e l}, \sigma_{\text {inel }}$ at $\sqrt{s}=7$ and $8 \mathrm{TeV}$. Measurement from cosmic rays, other LHC experiments and lower energy data are also superimposed together with the best fits from the COMPETE collaboration [6]. Right: comparison of the TOTEM measurement $\sigma_{t o t}$, $\sigma_{e l}, \sigma_{\text {inel }}$ obtained with different analysis methods (see text for more details).

\section{Single-diffractive and double-diffractive cross sections}

Preliminary measurements of the double and single diffractive cross section were obtained by using low pile-up runs at $7 \mathrm{TeV}$. The double diffractive cross section was obtained from a run triggered with $\mathrm{T} 2$, requiring at least one track in both $\mathrm{T} 2$ hemispheres and no tracks in $\mathrm{T} 1$.

The measurement is corrected in order to be representative for double diffractive events with central rapidity gap starting from $4.7<\left|\eta_{\min \pm}\right|<6.5$. This corresponds to the the cross section for DD events where both diffractive systems have masses between 3.4 and $8 \mathrm{GeV}$.

The non diffractive (ND) background was estimated by scaling the MC predictions according to a control sample from data dominated by ND (both sides of T1 and T2 detectors with tracks). The single diffractive (SD) background was estimated completely from the data using a SD dominated control sample (tracks in one T2 hemisphere, veto for tracks in the other T2 hemisphere and in T1), with a proton in the RPs. The double diffractive cross section was found to be $\sigma_{D D\left(4.7<\left|\eta_{\min }\right|<6.5\right)}=$ $120 \pm 25 \mu$ b. The measurement was also repeated requiring smaller rapidity gaps. The results are reported in table 1.

The single diffractive cross section measurement was obtained in a high $\beta^{*}=90 \mathrm{~m}$ run triggered with T2, requiring only 1 proton in the RPs. Depending on the different configurations of the inelastic telescopes, different diffractive mass ranges can be selected (see table 2). The $|t|$-differential diffractive cross sections was also measured for the different mass ranges. The distributions have been fitted with an exponential, $A \exp (-B|t|)$. The B parameter was found to be 
Table 1: Double diffraction cross sections for different sizes of the central rapidity gap.

\begin{tabular}{|l|l|l|}
\hline & $-4.7>\eta_{\min -}>-5.9$ & $-5.9>\eta_{\min -}>-6.5$ \\
\hline $4.7<\eta_{\min +}<5.9$ & $66 \pm 19 \mu \mathrm{b}$ & $27 \pm 5 \mu \mathrm{b}$ \\
\hline $5.9<\eta_{\min +}<6.5$ & $28 \pm 5 \mu \mathrm{b}$ & $12 \pm 4 \mu \mathrm{b}$ \\
\hline
\end{tabular}

Table 2: Single diffraction measurements for different ranges of the diffractive mass ("opposite" refers to the side of the IP with respect to the proton in the RP). The SD cross-section numbers contain two contributions: the leading proton can be reconstructed both in the left or in the right side of the IP.

\begin{tabular}{ccccc}
\hline SD class & Inelastic telescopes configuration & Mass & $\sigma_{S D}( \pm 20 \%)$ & $\mathrm{B}( \pm 15 \%)$ \\
\hline Low Mass & $\mathrm{p}+\mathrm{T} 2$ opposite only (no T1) & $3.4-8 \mathrm{GeV}$ & $1.8 \mathrm{mb}$ & 10.1 \\
Medium Mass & $\mathrm{p}+\mathrm{T} 2$ opposite + T1 opposite & $8-350 \mathrm{GeV}$ & $3.3 \mathrm{mb}$ & 8.5 \\
High Mass & $\mathrm{p}+\mathrm{T} 2$ opposite + T1 same & $0.35-1.1 \mathrm{TeV}$ & $1.4 \mathrm{mb}$ & 6.8
\end{tabular}

larger for smaller diffractive masses. Integrating each distribution down to $t=0$, the cross section was also determined for each single diffractive mass category $\left(\sigma_{S D}\right)$. The values of $B$ and $\sigma_{S D}$ are reported in table 2.

An optimal measurement of the diffractive mass (M) is achieved for low and medium masses using the estimation of the rapidity gap $\Delta \eta$ based on the charged track position in T1 and T2 $\left(\Delta \eta=-\ln \left(\mathrm{M}^{2} / s\right)\right.$, where $s$ is the Mandelstam variable). At high masses the proton $\xi=\frac{\Delta p}{p}$ measured by the RPs was instead used to determine the mass $\left(\mathrm{M}^{2}=s \xi\right)$.

\section{Forward multiplicities}

A preliminary measurement of the charged particle pseudorapidity density $(\mathrm{dN} / \mathrm{d} \eta)$ in $\mathrm{T} 2$ was obtained for inelastic events by using a low-luminosity run at $\sqrt{s}=8 \mathrm{TeV}$. The analysis is similar to the one implemented for the measurement at $7 \mathrm{TeV}$ [10], despite several improvements in the event selection, detector simulation and alignment were introduced. Now that the TOTEM trigger signal is able to go into the CMS trigger menu, the T2 minimum bias trigger was used also to trigger the readout of CMS and a simultaneous measurement of the $\mathrm{dN} / \mathrm{d} \eta$ on the same events was performed both in the CMS central tracker $(|\eta|<2.4)$ and in the very forward pseudorapidity region with T2 $(5.3<|\eta|<6.4)[11,12]$. The measurement is obtained for an "inclusive" inelastic sample of events, where at least a reconstructed track in $\mathrm{T} 2$ is required. The measurement is then corrected in order to be representative for inelastic events with at least a charged particle generated in the T2 $\eta$-acceptance with $P_{T}>40 \mathrm{MeV} / \mathrm{c}$. This selection guarantees that a fraction $>94 \%$ of the inelastic events is included in the sample. The pseudorapidity density measurement is also repeated for a non-single-diffractive enhanced sample where both side of $\mathrm{T} 2$ was required to have some tracks reconstructed. This measurement is then corrected in order to be representative for inelastic events with at least a charged particle generated in each side of the T2 $\eta$-acceptance, with $P_{T}>40 \mathrm{MeV} / \mathrm{c}$. A measurement of the charged particle $\mathrm{dN} / \mathrm{d} \eta$ for a single-diffactive enhanced 
sample is also ongoing. The results of the CMS and TOTEM $\mathrm{dN} / \mathrm{d} \eta$ analysis obtained with the inclusive sample, are reported in fig. 4.

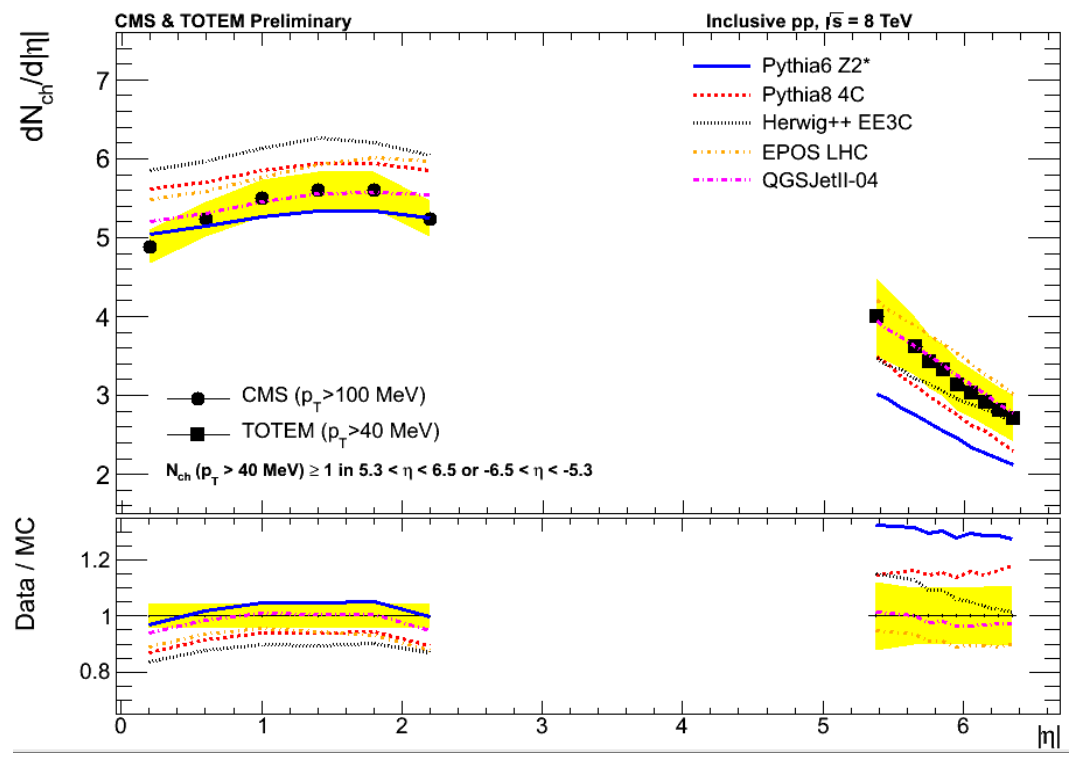

Figure 4: Charged particle $\mathrm{dN} / \mathrm{d} \eta$ obtained with a common CMS-TOTEM trigger. The measurement is representative of inelastic events with at least a charged particle generated in T2 $\left(P_{T}>40 \mathrm{MeV} / \mathrm{c}\right)$.

Having the two experiments different $\mathrm{P}_{T}$ acceptance the measurement includes charged particles with $P_{T}>100 \mathrm{MeV} / \mathrm{c}$ in the central tracker, while particles with $P_{T}>40 \mathrm{MeV} / \mathrm{c}$ are included in the T2 measurement. Consistently with the $P_{T}$ thresholds used in the analysis, the MC predictions are superimposed to the experimental points using different charged particle minimum $P_{T}$, depending on the pseudorapidity region.

\section{References}

[1] V. Berardi et al. (TOTEM Collaboration), CERNLHCC- 2004-002, CERN-LHCC-2004-020 (2004).

[2] M. Albrow et al. (The CMS and TOTEM DFP-WG), CERN-LHCC-2006-039/G-124 (2006).

[3] G. Antchev et al. (TOTEM Collaboration), Europhys. Lett. 95, 41001 (2011).

[4] G. Antchev et al. (TOTEM Collaboration), Europhys. Lett. 96, 21002 (2011).

[5] G. Antchev et al. (TOTEM Collaboration), Europhys. Lett. 101, 21002 (2013).

[6] J.R. Cudell et al. (COMPETE Coll.), Phys. Rev. Lett. 89, 201801 (2002).

[7] G. Antchev et al. (TOTEM Collaboration), Europhys. Lett. 101 (2013) 21003.

[8] G. Antchev et al. (TOTEM Collaboration), Europhys. Lett. 101 (2013) 21004.

[9] G. Antchev et al. (TOTEM Collaboration), CERN-PH-EP-2012-354 (to be published in Phys. Rev. Lett.).

[10] G. Antchev et al. (TOTEM Collaboration), Europhys. Lett. 98 (2012) 31002.

[11] G. Antchev et al. (TOTEM Collaboration), CERN-TOTEM-NOTE-2013-001.

[12] The CMS Collaboration, CMS Physics Analysis Summary, FSQ-12-026, 2013. 